\title{
L'institutionnalisme centré sur les acteurs
}

\author{
In: Politix. Vol. 14, №55. Troisième trimestre 2001. pp. 95-123.
}

\section{Article by an MPIfG researcher}

Renate Mayntz, Fritz W. Scharpf: L'institutionnalisme Centré sur les Acteurs. In: Politix 14(55), 95-123 (2001). Persée

The original publication is available at the publisher's web site: http://dx.doi.org/10.3406/polix.2001.1174

\section{Abstract}

The Actors Centred Instutionalism

Renate Mayntz et Fritz W. Scharpf

This article summarizes an approach that has been characteristic of research, carried out at the Max Planck Institute for the Study of Societies, Cologne, on the interaction between government policy and the self-organization of industrial and service sectors. According to this approach, outcomes are explained by the interactions of individual, collective and (primarily) corporate actors, all of them guided by their respective cognitive and normative orientations. In this context, institutions play a dual role. On the one hand, they define the rules and actor constellations within which interactions take place, and on the other hand they shape the normative and, to a lesser extent, the cognitive orientations of the actors involved. In combination, these institutional effects increase mutual predictability among the actors themselves and, by the same token, they create ontological preconditions which allow for limited generalization in the social sciences.

\section{Résumé}

L'institutionnalisme centré sur les acteurs

Renate Mayntz et Fritz W. Scharpf

Cet article résume une approche caractéristique de la recherche menée à l'Institut Max Planck pour les sciences sociales de Cologne, sur les interactions entre politique gouvernementale et autoorganisation des secteurs industriels et des services. Selon cette approche, les résultats sont expliqués par les interactions entre acteurs individuels, personnes collectives et (le plus souvent) corps constitués, tous étant guidé par leurs orientations cognitive et normative. Dans ce contexte, les institutions jouent un rôle double. D'un côté, elles définissent les règles et les constellations d'acteurs dans lesquels les interactions se déroulent; d'un autre côté, elles configurent les orientations normatives et, dans un moindre mesure, les orientations cognitives des acteurs impliqués. En se combinant, ces effets institutionnels augmentent la prévisibilité réciproque pour les acteurs eux-mêmes et, dans le même temps, créent les préconditions ontologiques qui permettent une généralisation limitée en sciences sociales.

Citer ce document / Cite this document :

Mayntz Renate, Scharpf Fritz W. L'institutionnalisme centré sur les acteurs. In: Politix. Vol. 14, N55. Troisième trimestre 2001. pp. 95-123.

doi : 10.3406/polix.2001.1174

http://www.persee.fr/web/revues/home/prescript/article/polix_0295-2319_2001_num_14_55_1174 


\section{L'institutionnalisme centré sur les acteurs*}

Renate MAYNTZ

Fritz W. SCHARPF

$\mathbb{Q} \mathrm{U}$

ne approche analytique s'appuie sur un canevas de catégories assez générales, qui servent à recenser et à classifier les faits empiriques et sont généralement adaptées à un type donné d'objets explicatifs. Dans le cadre de la thématique esquissée dans le premier chapitre, nous disposions de plusieurs approches de ce type, généralement associées à une théorie spécifique (par exemple la théorie de la différenciation, la théorie de la gouvernance, la théorie des jeux), dont aucune n'était cependant adaptée en soi aux spécificités de notre objet explicatif. Il a donc fallu, pour répondre correctement à la problématique, recourir à des éléments provenant de plusieurs théories, susceptibles d'éclairer différents aspects d'un faisceau de conditions complexes. Progressivement, nous avons pu dégager diverses questions dominantes et catégories analytiques, particulièrement utiles, qu'il a fallu ensuite ajuster les unes aux autres, au prix de grands efforts et de longues discussions. Nous allons décrire dans ce chapitre le résultat provisoire de ces efforts pour définir une approche "sur mesure » de la problématique de la régulation et de l'autoorganisation dans des sousensembles sociaux entiers, approche que nous avons appelée "institutionnalisme centré sur les acteurs". On gardera toutefois à l'esprit qu'une approche s'appuie sur des prémisses orientées et théoriques, mais

\footnotetext{
* Ce texte est la traduction du chapitre 2 "Der Ansatz des akteurzentrierten Institutionalismus " de l'ouv'rage dirigé par R. Mayntz et F.W. Scharpf, Gescllschaftliche Selbstregelung und politische Stenerung, Francfort/Main, Campus, 1995, p. 39-72. Elle a été réalisée par S. Avril de l'INISTCNRS et revue par $O$. Giraud et $P$. Laborier.
}

Politix. Volume $14-\mathrm{n}^{\circ} 55 / 2001$, pages 95 à 123 
qu'elle ne représente pas en soi une théorie sur le fond, rapportée à un objet. L'institutionnalisme centré sur les acteurs n'est donc pas un modèle apportant une explication, mais tout au mieux une heuristique de recherche, qui attire l'attention sur certains aspects de la réalité.

\section{La renaissance de l'institutionnalisme}

Le concept d'institutions connaît depuis quelque temps un regain d'intérêt. Les institutions ont toujours joué un rôle important en sciences sociales, sans que le concept ait toutefois été toujours utilisé de manière homogène. Herbert Spencer a décrit en sociologie une série d'institutions fondamentales correspondant à des besoins fondamentaux. Dans le prolongement de cette approche, le concept a pris une place centrale dans le fonctionnalisme en anthropologie culturelle et en sociologie, les institutions étant rapportées soit aux besoins fondamentaux de l'homme, soit à des impératifs systémiques. L'institution était également un concept essentiel pour Durkheim, qui mettait toutefois moins l'accent sur la fonctionnalité que sur l'élément de contrainte sociale externe influençant le comportement humain. Dans la théorie politique, les institutions occupent depuis l'Antiquité une place importante, voire prédominante ${ }^{1}$. Quant à la science politique, même après avoir abandonné la description comparée des institutions définies juridiquement (gouvernement, parlement, administration et partis) pour se tourner vers l'étude des comportements, elle s'est concentrée sur l'analyse de l'action dans les institutions politiques observées empiriquement ${ }^{2}$.

Le concept d'institution est donc appliqué tant à des construits sociaux qu'à des modèles de comportements normalisés ${ }^{3}$. Les tentatives visant à ramener le concept $d$ 'institution à des règles abstraites (mais applicables, autrement dit approuvées socialement si nécessaire ${ }^{4}$ ) et à le remplacer, pour les

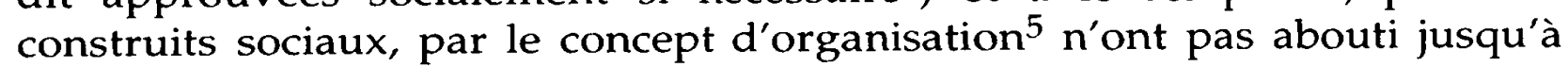
présent. Il est cependant généralement admis que, dans le concept d'institution, on insiste surtout sur les aspects de régulation se rapportant avant tout à la répartition et l'exercice du pouvoir, à la définition des

1. Cf. les contributions dans Göhler (G.), ed., Politische Institutionen im gesellschaftlichen Umbruch : Ideengeschichtliche Beiträge zur Theorie Politischer Institutionen, Opladen, Westdeutscher Verlag, 1990.

2. Notamment l'article de K. von Beyme dans Göhler (G.) et al., eds, Die Rationalität politischer Institutionen, Baden-Baden, Nomos, 1990.

3. Vanberg (V.), Markt und Organisation, Tübingen, Mohr, 1982, p. 32.

4. Pour R. Jepperson le coeur du concept d'institution est la représentation d'un "schéma stable pour des séquences d'activités répétées de façon chronique ", Jepperson (R.), "Institutions, Institutional Effects, and Institutionalism ", in Powell (W.), DiMaggio (P.), eds, The New Institutionalism in Organisational Analysis, Chicago, University of Chicago Press, 1991, p. 145.

5. Cf. North (D.), «A Transaction Cost Theory of Politics ", Journal of Theoretical Politics, 2, 1990. 
compétences, à la disposition de ressources ainsi qu'aux relations d'autorité et de dépendance.

Les institutions ont retrouvé, depuis peu, une place de choix dans l'institutionnalisme économique, la sociologie institutionnelle des organisations, le néo-institutionnalisme en science politique. Ces théories ont en commun de porter un regard critique sur certaines approches employées jusqu'à présent, mais cette critique porte sur un élément différent dans chaque cas et le concept d'institutions utilisé n'est pas le même.

En science économique, on distingue - bien que conceptuellement la distinction ne soit pas toujours aussi nette - une économie institutionnelle, qui cherche des explications institutionnelles aux situations économiques, et un institutionnalisme économique qui tente d'expliquer les institutions par des arguments économiques (autrement dit, comme le résultat d'une action rationnelle, calculée, émanant d'individus ${ }^{6}$ ). Le premier courant dénonce une théorie économique qui se contente, au niveau micro-économique, de l'analyse du comportement individuel rationnel et, au niveau macroéconomique, des variables agrégées de l'offre et de la demande, sans prêter attention aux particularités des structures de production et du marché, ni du cadre social dans lequel l'action économique s'insère. Ce n'est pas un hasard si des sociologues comme Granovetter ${ }^{7}$ et Streeck ont critiqué de telles omissions. Streeck a d'ailleurs contribué, par ses travaux empiriques, à l'élaboration d'une "théorie institutionnelle de l'offre dans les sociétés capitalistes avancées ${ }^{8}$ ». Bien que la théorie économique des institutions fondée sur une argumentation génétique ne s'écarte pas de l'approche néo-classique, elle critique cependant l'hypothèse d'un Homo œconomicus hyper-rationnel mais déjà domestiqué par les normes. Elle suppose qu'il existe d'une part des acteurs qui ne sont pas totalement rationnels, et d'autre part la possibilité toujours présente de préjudices "opportunistes». Ces deux éléments se traduisent par des " coûts de transaction", susceptibles de nuire à des échanges et coopérations normalement bénéfiques. L'existence d'institutions adéquates peut accroître la fiabilité des attentes mutuelles et réduire ainsi les coûts de transaction. Les avantages ainsi obtenus en terme d'efficience expliquent, selon les arguments tirés de théories évolutionnistes, non seulement le choix entre « le marché et la hiérarchie ", mais aussi le choix entre différentes formes d'organisation de I'entreprise?

6. Göhler (G) et al., eds, Die Rationalität politischer Institutionen, op. cit.

7. Granovetter (M), "Economic Action and Social Structure : The Problem of Embeddedness », American Journal of Sociology, 91, 1985.

8. Streeck (W.), Social Institutions and Economic Performance, Londres, Sage, 1992.

9. Williamson (O.), Markets and Hierarchies. Analysis and Antitrust Implications, New York, Free Press, 1975 ; Williamson (O.), The Economic Institutions of Capitalism. Firms, Markets, Relational 
L'institutionnalisme de la sociologie des organisations dénonce, quant à lui, la conception selon laquelle: «Les organisations étaient envisagées principalement comme des systèmes de production et/ou d'échange et leurs structures comme étant modelées, dans une large mesure, par leurs technologies, leurs transactions, ou les relations de pouvoir-dépendance qui résultent de ces interdépendances ${ }^{10}$. "Zucker détache la nouvelle " théorie institutionnelle " de toutes les approches fondées sur "l'hypothèse selon laquelle le comportement est motivé par les intérêts des acteurs humains et s'explique par ceux-ci ${ }^{11}$. » Il se réfère ici moins à Selznick - qui soulignait déjà, dans son étude sur la Tennessee Valley Authority ${ }^{12}$, que les organisations peuvent certes être créées comme des instruments, mais qu'elles acquièrent en général une valeur propre pour leurs membres et pour les acteurs intervenant dans leur environnement - qu'au constructivisme sociologique ou à l'interactionnisme symbolique. Dans l'ouvrage-clef de Berger et Luckmann, l'institutionnalisation est le processus par lequel les individus élaborent une définition commune de la réalité sociale; les règles vécues «tenues pour acquises", les représentations et les croyances jouent alors un rôle de premier plan ${ }^{13}$. En conséquence, l'institutionnalisme de la sociologie des organisations s'intéresse surtout aux éléments symboliques et cognitifs de l'environnement d'une organisation (mythes, traditions orales, idéologies légitimes, etc.) qui influencent celle$\mathrm{ci}^{14}$. Le concept d'institution est alors employé dans un sens très large et concret, synonyme de "culture ${ }^{15}$ ".

L'un des courants même du néo-institutionnalisme en science politique se fonde sur la critique des approches réductionnistes et utilitaristes, qui expliquent les phénomènes politiques comme les effets agrégés de l'action intéressée d'individus et n'accordent aucune importance aux structures organisationnelles ni aux actions à visée normative voire symbolique. Cette critique est formulée de manière incisive par March et Olsen, qui

Contracting, New York, Free Press, 1985 ; North (D.), Structure and Change in Economic History, New York, W.W. Norton, 1981.

10. Scott (R.), "The Adolescence of Institutional Theory ", Administration Science Quarterly, 32, 1987, p. 507.

11. Zucker (L.), ed., Institutional Patterns and Organization,. Cambridge, Ballinger, 1988, p. 4.

12. Selznick (P.), TVA and the Grass Roots. A Study in the Sociology of Formal Organization, New York, Harper \& Row, 1966.

13. Berger (P.), Luckmann (T.), Die gesellschaftliche Konstruktion der Wirklichkeit, Francfort/Main, Fischer, 1972.

14. Cf. les contributions dans Powell (W.), DiMaggio (P.), eds, The New Institutionalism in Organizational Analysis op. cit.

15. Cf. aussi la caractérisation synthétique de R. Jepperson : “ Dans l'analyse organisationnelle, en particulier, de nombreux commentateurs associent d'une façon ou d'une autre les institutions à la "culture", c'est-à-dire aux effets normatifs, aux idées, aux conceptions, aux "institutions préconscientes", aux mythes, aux rituels, à l'idéologie, aux théories ou aux récits" (Jepperson (R.), "Institutions, Institutional Effects, and Institutionalism», in Powell (W.), DiMaggio (P.), eds, The New Institutionalism..., op. cit., p. 150). 
s'intéressent à "la place des institutions en politique ${ }^{16}$ " et insistent sur l'importance de "comportements appropriés ", à visée normative, ainsi que des rituels, des cérémonies et des mythes. Ce courant du néoinstitutionnalisme en science politique rejoint ainsi l'institutionnalisme de la sociologie des organisations.

Un second courant du néo-institutionnalisme politique fait davantage appel à une notion déjà ancienne, qui entend par institutions politiques, les organes politiques centraux, autrement dit (contrairement à l'orientation sociologique organisationnelle décrite plus haut) des construits sociaux précis. Il ne s'agit cependant pas d'un simple retour à la pensée classique, encore que ce nouvel institutionnalisme soit apparu aux Etats-Unis avec la devise "bringing the state back $i^{17}$ ". Ce courant du néo-institutionnalisme politique, fondé sur la théorie de l'Etat, s'oppose aux approches béhavioristes ${ }^{18}$, structuralistes, et systémiques, qui ne s'intéressaient pas aux particularités de l'organisation politique (comme construction et systèmes de règles) et se concentraient soit sur les comportements individuels pertinents sur le plan politique, soit sur les effets des structures de pouvoir et d'intérêts sur les décisions politiques. Partant de là, Keck identifie une « renaissance de la vision institutionnelle ", accordant davantage d'attention aux structures organisationnelles du système politique. Ces dernières ne constituent pas une scène neutre, mais restreignent le champ d'action ou bien ouvrent de nouvelles options d'action. "Contrairement aux conceptions antérieures de la théorie de l'influence, les intérêts sociaux dominants... n'ont pas de répercussions directes sur les résultats du processus politique: ils sont modifiés ou filtrés par la machinerie du système politique ${ }^{19}$. " L'ouvrage collectif Do Institutions Matter? est un exemple éloquent de ces nouvelles tendances : ses coordinateurs cherchent à comprendre comment différents "arrangements institutionnels" influencent « la stabilité et l'efficacité des gouvernements ${ }^{20}$ ".

16. March (J.), Olsen (J.), "The New Institutionalism : Organizational Factors in Political Life ", American Political Science Review, 78, 1984, p. 735.

17. Evans (P.) et al., eds, Bringing the State Back In. Cambridge, Cambridge University Press, 1985.

18. Courant dans lequel K. von Beyme se distingue particulièrement. Cf. son article dans Göhler (G.), ed., Grundfragen der Theorie Politischer Institutional. Forschungsstand, Problem, Perspektiven, Opladen, West-deutscher Verlag, 1987.

19. Keck (O.), "Der neue Institutionalismus in der Theorie der Intemationalen Politik ", Politische Vierteljahresschrift, 32, 1991, p. 637.

20. Weaver (R.), Rockman (B.), eds, Do Institutions Matter? Government Capabilities in the United States and Abroad, Washington, DC, Brookings Institution, 1993, p. 4. 


\section{L'institutionnalisme centré sur les acteurs}

L'approche de l'institutionnalisme centré sur les acteurs, élaborée pour un objet d'étude spécifique, se rattache à ce dernier courant du néoinstitutionnalisme en science politique, dont elle se distingue toutefois par plusieurs aspects. En effet, ce courant ne se limite pas aux institutions politiques, il se base sur un concept étroit des institutions, considère celles-ci comme des variables pouvant être dépendantes aussi bien qu'indépendantes, et ne leur attribue pas d'effet déterminant. Au contraire, les facteurs institutionnels constituent bien plus un contexte de l'action, stimulant, potentialisant ou limitant. La figure 1 tente de schématiser cette approche. Si l'on veut étudier la régulation sectorielle et l'autorégulation dans les secteurs para-étatiques et leurs résultats, il est nécessaire de s'intéresser en priorité aux interactions entre les acteurs corporatifs, ces derniers pouvant être provisoirement définis comme des organisations capables d'action. Si l'accent est mis sur les acteurs corporatifs, c'est à la fois en raison du niveau d'analyse choisi (sous-systèmes sociaux) et de l'organisation très poussée propres aux secteurs dits para-étatiques; sans cela, on ne pourrait pour ainsi dire pas parler d'autoorganisation au niveau macrosocial. Bien que l'action des acteurs corporatifs occupe effectivement une place de premier plan dans notre champ d'investigation et notre problématique, cela ne signifie pas pour autant que nos tentatives d'explication se limitent à l'action de ces seuls acteurs. Même dans les secteurs très organisés, il arrive que l'action d'individus, au niveau microsocial, contribue de manière décisive à la situation que l'on souhaite expliquer ${ }^{21}$. En outre, les stratégies d'acteurs corporatifs peuvent aussi s'expliquer, dans certains cas, par l'action, non déterminée par les institutions, d'individus en leur qualité de membres, de titulaires d'une fonction ou de représentants. Ceci implique à nouveau, en principe, une perspective multi-niveaux, dans laquelle le cadre institutionnel influence l'action des organisations, ces dernières créant à leur tour le cadre institutionnel déterminant l'action de leurs membres.

Dans les secteurs sociaux très organisés, chaque acteur corporatif fait en général partie d'une constellation d'acteurs plus ou moins complexe. De fait, en science politique, le néo-institutionnalisme s'intéresse moins aux différentes institutions politiques qu'à l'effet que produisent certains dispositifs institutionnels, que l'on cherche à styliser de manière à en extraire des types idéaux, puis à spécifier selon leur mode de fonctionnement. On peut citer comme exemple le "modèle de démocratie parlementaire de Westminster" ou le « fédéralisme unifié à l'allemande » (Verbundföderalismus). Ces catégories définies dans une optique phénoménologique orientée vers les formes ont

21. Cf. Schimank (U.), Wasem (J.), «Die staatliche Steuerbarkeit unkoordinierten kollektiven Handelns", in Mayntz (R.), Scharpf (F.W.), eds, Gesellschaftliche Selbstregelung und politische Steuerung, Francfort/Main, Campus, 1995. 
toutefois l'inconvénient d'être assez peu abstraites et de ne concerner que les structures de décision politiques. Dans l'institutionnalisme centré sur les acteurs, en revanche, on s'efforce de définir des catégories analytiques permettant d'identifier les aspects théoriques pertinents des constellations $d^{\prime}$ acteurs englobant tout un secteur. C'est la raison pour laquelle, on ne peut pas se limiter aux institutions (ou acteurs) politiques: il est nécessaire d'intégrer tous les acteurs pertinents dans les différents champs de régulation sociale. On évite ainsi le "point de vue du législateur", commun à bien des études en science politique, dans lesquelles les champs de régulation sociale constituent un environnement plus ou moins amorphe et passif. Au contraire, l'accent est mis ici sur l'intégration des acteurs étatiques et non étatiques dans les structures qui influencent leur action.

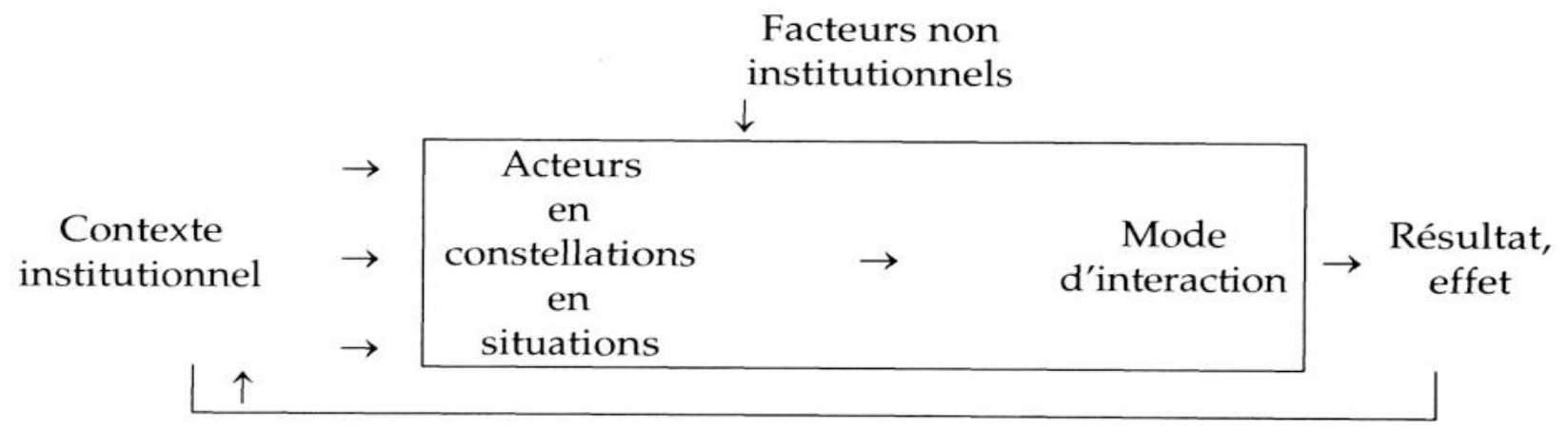

Figure 1. Vue d'ensemble du modèle analytique

A la différence des tentatives d'élargissement "culturaliste», le concept d'institutions dans l'institutionnalisme centré sur les acteurs est relativement étroit et se concentre sur les aspects de régulation. Cette orientation analytique, fondamentale pour l'ensemble de la démarche, a deux conséquences importantes. $D^{\prime}$ une part, les institutions ne sont pas interprétées simplement comme le résultat d'un développement évolutionniste et tenues pour acquises: elles peuvent être volontairement modelées et modifiées par l'action d'acteurs identifiables. L'approche centrée sur les acteurs considère donc les institutions comme des variables aussi bien dépendantes qu'indépendantes. D'autre part, en limitant le concept d'institution aux aspects de régulation, on met en valeur une prémisse, souvent simplement citée de manière formelle, selon laquelle le contexte institutionnel autorise ou entrave l'action, mais ne la détermine pas. Bien qu'elles s'en défendent, les approches institutionnalistes sont souvent crypto-déterministes concernant l'action des acteurs, surtout lorsque le concept d'institutions est assez large pour englober non seulement les normes du comportement adéquat, mais aussi les éléments cognitifs et symboliques influençant l'action. Si l'on inclut encore dans ce concept les pratiques de la vie quotidienne, sans se poser la question de leur provenance, les acteurs sont alors privés de toute latitude et il 
ne reste au sujet que quelques impulsions idiosyncrasiques, quasiment inaccessibles à l'analyse sociologique théorique. Cette conceptualisation est peut-être exploitable dans les théories sociologiques qui s'intéressent au comportement individuel (moyen). En revanche, l'explication des processus de régulation et d'autoorganisation au niveau macrosocial nécessite quant à elle un concept plus étroit des institutions, permettant de considérer l'action des acteurs comme une variable autonome et d'analyser ainsi des situations où, malgré un cadre institutionnel fondamentalement inchangé, on observe des modifications lourdes de conséquences au niveau de l'action ${ }^{22}$.

L'analyse des structures sans référence aux acteurs est tout aussi déficiente que l'analyse de l'action des acteurs sans référence aux structures, comme Alexander l'a rappelé récemment encore ${ }^{23}$. L'institutionnalisme centré sur les acteurs surmonte cette dichotomie par sa double perspective sur les acteurs et les institutions. La démarcation explicite, ainsi proposée, entre institutions et action observable, rejoint ainsi le débat de longue date sur les rapports entre acteur et système ${ }^{24}$ ou structure et agency ${ }^{25}$. Ce débat porte toutefois davantage sur le primat de la structure ou de l'action de l'acteur que sur l'intégration de ces deux perspectives. De même, la démarcation entre structure et agency reste floue dans l'analyse ${ }^{26}$. Le concept d'agency est associé à celui de libre arbitre, mais dans la réalité, les acteurs sont toujours marqués par des influences sociales. Cela ne pose pas de problème si l'on distingue les règles institutionnelles de l'action des acteurs réels, comme le fait l'institutionnalisme centré sur les acteurs 27 .

Dans les explications proposées dans le cadre de l'institutionnalisme centré sur les acteurs, l'action observable des acteurs a toujours une fonction de "cause première" (proximate cause), tandis que le cadre institutionnel représente pour nous la "cause seconde " (remote cause) centrale. Entre ces deux pôles, de nombreux facteurs interviennent pour codéterminer les acteurs et leurs orientations, leurs relations et les situations dans lesquelles

22. Par exemple les changements de stratégie politico-économique de ces vingt dernières années. Le succès de la réforme Seehofer dans le secteur de la santé, après l'échec des multiples tentatives précédentes, ne s'explique pas non plus par une modification des conditions institutionnelles.

23. Alexander (J.), « Commentary : Structure, Value, Action ", in Hamilton (P.), ed., Talcott Parsons : Critical Assessment, Londres, Routledge, 1992.

24. Crozier (M.), Friedberg (E.), L'acteur et le systeme, Paris, Seuil, 1977 ; Weyer (J.), « System und Akteur. Zum Nutzen zweier soziologischer Paradigmen bei der Erklarung erfolgreichen Scheitems ", Kölner Zeitschrift für Soziologie und Sozialpsychologie, 45, 1993.

25. Par exemple Giddens (A.), The Constitution of Society. Outline of a Theory of Structuration, Cambridge, Polity Press, 1984.

26. Alexander (J.), Sowie Differenzierung und kultureller Wandel, Francfort/Main, Campus, p. 502.

27. Pour A. Giddens (The Constitution of Society..., op. cit., p. 282), les acteurs agissent surtout par habitude. La variance, qui parvient à ramener un comportement globalement habituel dans des explications du reste déterminées structurellement, est pourtant si faible que le résultat de l'action paraît en pratique déterminé par la structure. 
ils interagissent. Ainsi, les acteurs corporatifs sont constitués, ou du moins approuvés, par la législation, mais cela ne suffit pas à déterminer entièrement leurs caractéristiques en tant qu'organisation sociale, ni l'orientation de leur action dans une situation concrète. Il en va de même des structures plus globales dans lesquelles ils s'insèrent. Ici encore, par exemple, une partie des relations durables est donnée par les institutions, mais une autre partie est plus informelle, voire parfois « hors la loi ». Enfin, les interactions entre certains acteurs sont aussi motivées par des règles institutionnelles, mais les situations réelles contiennent également de nombreux éléments non institutionnels. Nous détaillerons ci-après ces composantes de notre approche. Pour des raisons pragmatiques et pour assurer une meilleure transparence dans la recherche d'explications, nous n'employons aucune grille de catégories pour le recensement systématique des facteurs $d$ 'influence, y compris des facteurs non institutionnels.

\section{Le contexte institutionnel}

Une classification différentielle des contenus possibles de la régulation devient très vite trop complexe ${ }^{28}$ et finit par dépasser la systématique juridique du droit constitutionnel, administratif, fiscal, pénal, civil, du droit des sociétés, des associations, du travail ou du droit procédural. L'important est que les règles institutionnelles (définies, appliquées et approuvées) permettent de fonder la fiabilité des attentes mutuelles et rendent ainsi possible l'action sociale par-delà les limites des relations personnelles, comme l'ont souligné les institutionnalistes, à juste titre, par opposition à une théorie économique dépourvue de dimension institutionnelle ${ }^{29}$. Une classification minimaliste des contenus de la régulation, en vue de l'analyse des structures sectorielles et des processus de décision, doit tout d'abord distinguer, selon les définitions courantes des institutions, les règles qui :

- définissent les normes (matérielles) de comportement et de procédure (formelles) dans des situations données,

- autorisent ou au contraire refusent l'accès de certains groupes à des ressources financières, juridiques, personnelles, techniques ou naturelles, - et déterminent les relations (notamment de dominance et de dépendance) entre des acteurs donnés.

28. Cf. Ostrom (E.), "A Method of Institutional Analysis ", in Kaufmann (F.-X.) et al., dir., Guidance, Control, and Eialuation in the Public Sector. The Bielefeld Interdisciplinary Project, Berlin, De Gruyter, 1986 ; Ostrom (E.), "An Agenda for the Study of Institutions ", Public Choice, 48, 1986.

29. North (D.), "A Transaction Cost Theory of Politics ", art. cité ; North (D.), "Institutions ", Journal of Economic Perspectices, 5, 1991. 
Il est en outre important, dans notre optique, que les acteurs corporatifs soient constitués par des règles institutionnelles. Il est même fréquent qu'ils soient créés par une décision de l'Etat, ce qui leur confère, en une seule fois, une mission et des compétences ${ }^{30}$. Il convient enfin de souligner que, dans le cadre de la régulation institutionnelle des procédures, des motifs d'interaction entre certains acteurs sont définis et des arènes sont créées dans lesquelles des acteurs donnés se réunissent pour discuter ou décider de thèmes spécifiques, tout en étant subordonnés à des règles de décision déterminées. En définissant les tâches et les relations entre les acteurs, la régulation institutionnelle crée des structures de division du travail qui peuvent aussi être décrites par les concepts de la différenciation sociale. Bien que la théorie systémique, qui ne laisse pas de part aux acteurs et argumente uniquement sur la base des relations de communication, diffère fortement, dans sa perspective analytique, de l'institutionnalisme centré sur les acteurs, celui-ci se rapproche beaucoup d'une théorie de la différenciation mettant l'accent sur les structures sociales réelles ${ }^{31}$. Ainsi, la structure particulière du système de santé allemand, avec sa séparation caractéristique entre soins hospitaliers et soins ambulatoires ainsi que son mode de financement basé sur les cotisations obligatoires ${ }^{32}$, ou encore la proximité de l'enseignement et de la recherche dans les universités allemandes, avec les conséquences qu'elle entraîne ${ }^{33}$, constituent des formes de différenciation sociale modelées par les institutions. La théorie de la différenciation met l'accent à la fois sur un aspect important pour la description des structures sectorielles et des constellations d'acteurs (la division du travail) et sur une composante importante (fonctionnelle) de l'orientation de l'action.

\section{Acteurs et orientation de l'action}

Le cadre institutionnel, définissant les règles que l'on doit soi-même respecter et que l'on souhaite que les autres respectent, est un élément constitutif des acteurs et des constellations d'acteurs; il structure leur accès aux ressources nécessaires à l'action, influence leurs orientations et modèle des aspects importants des situations auxquelles chaque acteur est confronté. Pour autant, ce cadre institutionnel n'englobe pas tous les types d'action, ni tous les facteurs pertinents pour l'action, et ne détermine pas entièrement non plus l'action lorsqu'il entre en jeu. Cela signifie non

30. La capacité d'action des individus repose souvent, elle aussi, sur des conditions institutionnelles. S'agissant des personnes physiques, cela est toutefois moins évident et l'influence sur les comportements est moins déterminante.

31. Mayntz (R.), "Funktionelle Teilsysteme in der Theorie sozialer Differenzierung ", in Mayntz (R.) et al., eds, Differenzierung und Verselbständigung, Francfort/Main, Campus, 1988.

32. Alber (J.), Das Gesundheitswesen der Bundesrepublik Deutschland. Entwicklung, Struktur und Funktionsweise, Francfort/Main, Campus, 1992.

33. Schimank (U.), Hochschulforschung im Schatten der Lehre, Francfort/Main, Campus, 1995. 
seulement que l'on peut enfreindre les normes, abuser du pouvoir ou recourir à des interactions informelles, mais aussi que l'accès aux ressources n'est régulé que partiellement par les institutions, notamment s'agissant de ressources naturelles et techniques. Tous ces aspects sont évidents, si l'on s'intéresse non plus au cadre institutionnel mais aux acteurs qui agissent dans ce cadre.

\section{Les acteurs}

Selon March et Olsen ${ }^{34}$, le concept de «bringing the state back in " implique aussi que les institutions politiques soient considérées comme " des acteurs à part entière »: on peut noter, avec raison, que les institutions politiques peuvent être traitées comme des acteurs, de la même manière que l'on traite les individus comme des acteurs ${ }^{35}$. Cela va de soi si l'on désigne par institutions non seulement les systèmes de règles, mais aussi les formes sociales. Si l'on utilise en revanche un concept d'institutions étroit, centré sur les règles, il faut alors dans l'analyse faire la distinction entre institutions et acteurs. Les systèmes de règles "n'agissent » pas, mais ils peuvent être un élément constitutif des acteurs et modeler des caractéristiques importantes de ceux-ci. On peut dès lors considérer les structures sociales comme les organisations aussi bien du point de vue des règles qu'elles incarnent, autrement dit du point de vue institutionnel, que du point de vue de leur capacité d'action, autrement dit en tant qu'acteurs corporatifs.

Selon la définition de Coleman ${ }^{36}$, un acteur corporatif est un groupe organisé de façon formelle, majoritaire dans son sous-ensemble social capable d'action, et disposant pour agir de ressources centralisées (c'est-àdire qui ne sont plus disponibles au niveau individuel des membres), dont l'utilisation est déterminée soit par la voie hiérarchique (par exemple dans les entreprises ou les administrations), soit à la majorité (dans les partis ou les associations). La manière dont les organisations se voient habilitées à agir et à quelles conditions elles le sont, est un thème largement débattu en sociologie ${ }^{37}$. Les conditions internes aux organisations les plus importantes pourront être la capacité à former une volonté collective et le contrôle effectif

34. March (J.), Olsen (J.), "The New Institutionalism...", art. cité, p. 738.

35. Ibid., p. 742.

36. Coleman (J.), Poicer and the Structure of Society, New York, Norton, 1974.

37. Cf. Mayntz (R.), "Corporate Actors in Public Policy: Changing Perspectives in Political Analysis", Norsk Statsiitenskntelig Tidsskrift, 3, 1986 ; Schneider (V.), Werle (R.), "Vom Regime zum korporativen Akteur. Zur institutionellen Dynamik der Europaischen Gemeinschaft ", in Kohler-Koch (B.), ed., Regime in den intermationalen Beziehungen, Baden-Baden, Nomos, 1989 ; Flam (H.), Corporate Actors, MPIFG Discussion Paper 90/11, Cologne, MPI für Gesellschaftsforschung, 1990; Wiesenthal (H.), Unsicherheit und Multiple-Self-Identitat : Eine Spekulation iiber die Voraussetzungen strategischen Handelns, MPIFG Discussion Paper 90/2, Cologne, MPI fur Gesellschaftsforschung, 1990 ; etc. 
de l'action des membres de l'organisation; c'est sur ces critères que l'on évaluera si l'action peut être attribuée à l'organisation (et non pas à certains de ses membres). Il ne s'agit pas ici d'une alternative. Toute organisation n'est pas à tout moment dotée de la même capacité d'action, comme l'a montré par exemple la reconstitution des comportements de l'Académie des sciences de RDA au cours de l'unification allemande ${ }^{38}$. La capacité d'action des organisations est donc une variable.

Les acteurs individuels et corporatifs (organisations) sont liés par une relation d'inclusion: tous les acteurs corporatifs ont pour membres des acteurs individuels. Généralement, pourtant, lorsqu'on cherche à expliquer l'action stratégique des acteurs collectifs, on néglige les processus qui se déroulent au niveau microsocial, soit au niveau de leurs membres. Cette omission est principalement pragmatique: la complexité de nombreux processus ne peut être réduite à des proportions gérables que si l'on se concentre sur le comportement des acteurs corporatifs. Il en résulte toutefois une perte d'acuité. Les processus internes aux organisations sont en effet des déterminants importants de la perception des situations et des choix stratégiques de celles-ci. A ce titre, ils doivent être inclus dans l'analyse chaque fois que les facteurs institutionnels et le contexte de la situation ne suffisent pas à expliquer le comportement apparent d'un acteur corporatif. L'approche sur plusieurs niveaux, intégrant les acteurs individuels, est particulièrement précieuse lorsque l'on étudie les interactions entre acteurs corporatifs. Toujours pour des raisons de simplification, les acteurs individuels représentant une organisation sont généralement assimilés à l'acteur corporatif lui-même. Cela ne poserait aucun problème si tous les représentants étaient intégralement dirigés par un mandat impératif de leur organisation, ou si un seul représentant pouvait diriger parfaitement l'organisation. Or, il n'en est généralement rien. Les organisations sont le plus souvent des coalitions de groupes ayant des intérêts, des perceptions et des potentiels $d$ 'influence différents ${ }^{39}$ et les individus agissant pour le compte d'une organisation disposent presque toujours d'une certaine latitude d'action (qui peut être considérable), notamment parce qu'aucune organisation ne dispose de stratégies prédéfinies pour toutes les situations auxquelles elle est confrontée. De ce fait, l'issue de négociations peut être différente selon la personne qui représente l'organisation, selon qu'il s'agit d'un membre de l'encadrement, disposant d'une grande autonomie d'action et autorisé à engager et à discipliner l'organisation, ou par exemple d'un spécialiste d'un rang hiérarchique moins élevé. Pour la pratique de recherche, cela signifie en pratique qu'il faut éventuellement (mais pas

38. Mayntz (R.), "Academy of Sciences in Crisis : A Case Study of a Fruitless Struggle for Survival ", in Schimank (U.), Stucke (A.), eds, Coping With Trouble. How Science Reacts to Political Disturbances of Research Conditions, Francfort/Main, Campus, 1994.

39. Cyert (R.), March (J.), A Behavioral Theory of the Firm, Englewood Cliffs, Prentice-Hall, 1963. 
toujours), dans notre tentative d'explication de l'action d'un acteur corporatif, tenir compte aussi des orientations des individus agissant dans et pour les organisations.

Il ne suffit pas, pour décrire la structure de sous-systèmes sociaux ou de secteurs politiques entiers, d'utiliser seulement deux catégories d'entités sociales, à savoir les acteurs individuels et corporatifs. Les individus ne se regroupent pas seulement en organisations, mais aussi en familles, en réseaux de connaissances moins formels, en foules occasionnelles ou en simples "quasi-groupes", autrement dit dans des catégories de personnes ayant en commun une caractéristique précise et pertinente pour l'action. Ce sont eux, tous ensemble, qui peuvent jouer un rôle important dans les processus de régulation et d'autoorganisation et dans la production de macroeffets, qu'on leur concède ou non une capacité d'action. Les quasigroupes ne sont pas des acteurs capables d'action, mais ils sont souvent les destinataires de tentatives de gouvernance sélectives. Ceci étant dit, les individus composant un quasi-groupe peuvent parfois être modélisés en tant qu'acteurs, lorsque les individus rassemblés dans le quasi-groupe réagissent chacun de la même manière à un stimulus externe. Il en va de même lorsque les membres d'un quasi-groupe réagissent les uns aux autres, ce qui renforce par l'intérieur la réaction collective. Dans ces quasi-groupes, la dynamique propre de l'action convergente, renforcée par les interactions internes, joue un rôle important même dans les secteurs très organisés de la société. Elle seule peut expliquer le passage étonnamment rapide des soins médicaux ambulatoires en polyclinique à la médecine en cabinet de ville en Allemagne de l'Est $^{40}$. Si les orientations d'action des membres d'un tel mouvement social convergent consciemment, autrement dit s'ils aspirent à une action collective sans organisation formelle, on peut parler "d'acteur collectif". C'est dans ce sens, que l'on accorde aux classes sociales, par exemple, le statut d'acteur ${ }^{41}$. Qu'ils soient constitués ou simplement régulés par les institutions ou par des phénomènes extra-institutionnels, tous ces éléments sociaux (pris comme acteurs ou comme composante de leur contexte d'action) peuvent être importants pour les processus de gouvernance et d'autoorganisation.

\section{Orientations de l'action}

Les règles institutionnelles ont rarement une portée universelle. L'exploitation de la latitude qui subsiste en réalité est fortement déterminée

40. Wasem (J.), "Niederlassung oder "Poliklinik". Zur Entscheidungssituation der ambulant tatigen Ärzte im Beitrittsgebiet ", in Oberender (P.), ed., Stenerungsprobleme im Gesundheitswesen, Baden-Baden, Nomos, 1992 ; Schimank (U.), Wasem (J.), " Die staatliche Steuerbarkeit... ", art. cité. 41. Touraine (A.), The Self-Production of Society, Chicago, University of Chicago Press, 1977. 
par l'orientation de l'action par les acteurs concernés. L'orientation est ellemême en partie modelée par les institutions, notamment par les tâches ou les objectifs d'action fixés, mais aussi par la position des acteurs au sein de leur constellation. Dans le même temps, elle est également déterminée par les qualités des acteurs individuels ou corporatifs, indépendantes du contexte et liées à la socialisation ou à des facteurs historiques.

Cette vision des rapports entre les institutions et l'orientation de l'action n'est pas la même que celle proposée dans l'approche du « choix rationnel » (rational choice), qui considère les institutions, lorsqu'elles sont thématisées, comme des restrictions externes imposées à une maximisation des profits de nature égoïste et rationnelle. Elle diffère aussi du modèle de filtrage, dans lequel les institutions déterminent des "corridors d'action" à l'intérieur desquels les choix peuvent se faire de manière rationnelle ${ }^{42}$. L'opposition de deux orientations d'action, l'orientation normative imputée à l'institution et l'orientation rationnelle par rapport à un but (zweckrational) imputée à l'acteur, ne rend pas totalement compte de la réalité. Les acteurs n'utilisent pas uniquement la latitude que ménagent les règles institutionnelles pour maximiser leur profit. A l'inverse, il n'est pas rare que les acteurs considèrent comme "approprié", par rapport aux institutions, un comportement rationnel par rapport à un but, voire visant une maximisation égoïste de leur profit.

Lorsque l'on veut décrire les orientations de l'action, il est important $d$ 'identifier d'abord quelle est l'unité sociale dont on adopte la perspective pour envisager les différentes options s'offrant aux individus agissants (car, en dernière analyse, les acteurs corporatifs eux-mêmes n'agissent que par l'intermédiaire d'individus). En s'inspirant de la distinction formulée par Parsons entre « orientation par rapport à soi » et " orientation par rapport à la collectivité ", on peut établir une différenciation entre l'action centrée sur soi et l'action centrée sur le système ${ }^{43}$. Cette dernière mérite toutefois une différenciation plus fine. Les individus peuvent agir en tant que membre d'une classe sociale, d'un groupe ethnique, d'une organisation ou d'un Etat. Parce qu'ils appartiennent en général à plusieurs unités sociales hiérarchisées, des conflits sont possibles non seulement entre les actions centrées sur soi et les actions centrées sur le groupe, mais aussi dans les rapports à différents groupes: ainsi, les membres du Bundesrat allemand (Conseil fédéral), par exemple, peuvent, lorsqu'ils agissent, se placer dans la perspective de leur Land ou bien dans celle de leur parti. La détermination empirique du référent social dirigeant l'action n'a donc rien d'anecdotique.

42. Czada (R.), Windhoff-Héritier (A.), eds, Political Choice: Institutions, Rules, and the Limits of Rationality. Francfort/Main, Campus 1991.

43. Parsons (T.), The Social System, Glencoe, Free Press, 1951, p. 60. 
On peut dès lors distinguer, du point de vue du contenu, des aspects cognitifs et motivationnels dans l'orientation de l'action. Les orientations cognitives concernent la perception de la situation de l'action et de sa causalité, les options disponibles et les résultats escomptés. Leur importance pour la réussite de l'action est tout aussi évidente que la difficulté à les mesurer empiriquement ${ }^{44}$. L'issue de l'action dépend en particulier de l'existence et de l'ampleur d'une éventuelle différence entre la perception des acteurs et la réalité, de la manière dont les interprétations de la situation et les hypothèses sur la fin et les moyens sont intégrées dans des concepts stratégiques cohérents, la manière dont ceux-ci sont répartis selon les acteurs concernés dans des "communautés épistémiques ${ }^{45}$ » ou des «coalitions advocatives 46 » et enfin de la manière dont ils peuvent être modifiés par des processus d'apprentissage individuels et collectifs. Le sort qu'a subi l'Académie des Sciences de RDA au cours de l'unification allemande 47 montre bien qu'il n'est pas possible de fonder des stratégies de résolution des problèmes efficaces sur des perceptions inexactes (que, pour cette raison, la recherche empirique des cognitions gouvernant effectivement l'action est bien une condition nécessaire pour trouver une explication pertinente).

Une interprétation concordante de la situation peut être une condition nécessaire à l'action commune. Cependant, selon le phénomène universel de "perception sélective 48 ", il est vraisemblable que des acteurs indépendants ayant des missions, des attributions ou des intérêts différents perçoivent des fragments différents de la réalité avec une acuité différente. Il est donc peu probable qu'une constellation d'acteurs étatiques et non-étatiques interdépendants, confrontée à un problème commun, parvienne spontanément à une perception concordante de la situation, des options d'action de chaque intervenant et des effets escomptés de celles-ci. Au contraire, les "schémas cognitifs" des acteurs (cognitive maps) 49 seront plutôt divergents. Dans l'idéal, le processus de résolution du problème permet l'intégration de ces perspectives éclatées ${ }^{50}$. Si cela n'est pas possible,

44. Cf. Vowe (G.), Qualitative Inhaltsanalyse. Cognitive Mapping Policy Arguer. Demonstration der Vorgehensweise zur Analyse politischer Kognition. Forschungsbencht zum Projekt Handlungsorientierungen, manuscrit, Cologne, MPI fur Gesellschaftsforschung, 1993.

45. Haas (P.), "Introduction : Epistemic Communities and International Policy Coordination ", International Organisation, 46, 1992.

46. Sabatier (P.), "Knowledge, Policy-Oriented Learning, and Policy Change ", Knozledge : Creation, Diffusion, Utilization, 8, 1987.

47. Mayntz (R.), « Academy of Sciences in Crisis... », art. cité.

48. Dearborn (D.), Simon (H.), "Selective Perception: A Note on the Departmental Identification of Executives », Sociometry, 21, 1958.

49. Axelrod (R.), ed., Structure of Decision. The Cognitize Maps of Political Elites, Princeton, Princeton University Press, 1976.

50. Cohen (M.), "The Power of Parallel Thinking ", Journal of Economic Behaviour and Organization, 2, 1981 ; Quirk (P.), «The Cooperative Resolution of Conflict ", American Political Science Review, 83, 1989. 
il est tout de même important que les participants perçoivent au moins correctement les différentes interprétations de la situation en présence, et qu'ils puissent anticiper correctement les stratégies probables de chaque partie concernée ${ }^{51}$.

Les aspects motivationnels de l'orientation concernent les facteurs d'impulsion incitant à une action dotée de sens (ou dotée de sens, sinnhaft, au sens où l'entend Max Weber) ou plus, étroitement concernent, les considérations régissant le choix des options d'action (pour l'adapter à l'action stratégique des acteurs corporatifs). Si l'on ne peut faire l'impasse ni sur les émotions ni sur les habitudes aveugles dans l'explication des comportements individuels observés, l'étude de l'action des acteurs corporatifs peut souvent se concentrer sur les intérêts, normes et identités guidant l'action.

Le concept d'intérêt peut revêtir des significations très différentes ${ }^{52}$, et on a tendance à l'employer de façon assez floue même dans les analyses sociologiques. Aujourd'hui, c'est le sens du profit étroit et égocentrique qui domine. En conséquence, l'action motivée par l'intérêt est opposée à l'action fondée sur les normes ou les valeurs, et elle est facilement qualifiée péjorativement d'égoïste. C'est faire bien peu de cas de la connotation positive que peut avoir l'action autonome. Comme le rappelle Albert O. Hirschmann, dans les débats idéologiques des XVII ${ }^{e}$ et XVIII ${ }^{e}$ siècles, l'intérêt était considéré comme le pendant « raisonnable » de la passion, idiosyncrasique et destructrice $^{53}$. Cette acception ouvre la voie à une définition de l'intérêt utilisable pour l'analyse : puisqu'ils sont raisonnables, les intérêts peuvent être définis objectivement, au niveau abstrait, comme des impératifs fonctionnels, en recourrant (comme le fonctionnalisme sociologique) à des catégories biologiques évolutionnistes. Fondamentalement, les intérêts visent la survie à long terme : ce sont des objectifs d'action centrés sur le sujet et que celui-ci doit s'efforcer d'atteindre pour assurer sa propre survie. Le bien-être physique, la liberté d'action et l'accès aux ressources importantes, notamment, le pouvoir, la reconnaissance sociale et la possession d'un domaine protégé, peuvent être considérés comme des intérêts quasiment "standard" chez tous les êtres vivants. De la même manière, les acteurs corporatifs ont généralement aussi

51. C'est ainsi que la forte augmentation du chômage en Allemagne en 1974-1975 a été imputée au fait que les syndicats allemands, prisonniers de leur conception keynésienne, n'avaient pas perçu à temps le tournant monétariste pris par la Bundesbank, ou du moins ne l'avaient pas jugé crédible. Ce type de quiproquo a pu être évité en Autriche par une communication intensive entre la banque centrale et les partenaires sociaux. Cf. Scharpf (F.W.), Sozialdemokratische Krisenpolitik in Europa, Francfort/Main, Campus, 1987.

52. L'infinitif latin interesse (être important) domine dans le sens du français intérêt et de l'adjectif allemand interessant, tandis que le substantif allemand Interesse, apparu dans le jargon juridique au XIII' siècle, a le sens plus restreint d'utilité économique.

53. Hirschmann (A.), The Passions and the Interests. Political Arguments for Capitalism Before Its Triumph, Princeton, Princeton University Press, 1977. 
leurs propres intérêts, liés à leur propre existence, à leurs ressources et à leur autonomie ${ }^{54}$.

\begin{tabular}{|c|c|c|}
\hline & Intérêts & Normes \\
\hline $\begin{array}{l}\text { Déterminant } \\
\text { externe }\end{array}$ & $\begin{array}{c}\text { impératifs fonctionnels } \\
\text { । } \\
\text { sélection }\end{array}$ & $\begin{array}{c}\text { attentes normatives } \\
\text { । } \\
\text { sélection } \\
\end{array}$ \\
\hline $\begin{array}{l}\text { Orientation } \\
\text { durable } \\
\text { de l'action }\end{array}$ & $\begin{array}{c}\downarrow \\
\text { préférences stables } \\
\mid \\
\text { activation } \\
\end{array}$ & $\begin{array}{c}\downarrow \\
\text { normes internalisées } \\
\mid \\
\text { activation } \\
\end{array}$ \\
\hline $\begin{array}{l}\text { Motivations } \\
\text { liées à la } \\
\text { situation }\end{array}$ & $\begin{array}{c}\downarrow \\
\text { objectifs d'action } \\
\text { liés à la situation } \\
\text { (motifs «pour que ») }\end{array}$ & $\begin{array}{c}\downarrow \\
\text { causes de l'action } \\
\text { liées à la situation } \\
\text { (motifs « parce que ») }\end{array}$ \\
\hline
\end{tabular}

Figure 2. Niveaux d'orientation de l'action

Les intérêts standard imputables doivent d'abord être concrétisés avant de pouvoir diriger l'action. Ce sont alors les règles institutionnelles constitutives de l'acteur qui aident à définir ce que signifie l'autonomie dans un cas particulier ou à délimiter un domaine. Par exemple, ce sont le rôle social pour l'acteur individuel et l'objet de l'organisation pour l'acteur corporatif qui définissent l'éventail de tâches dans lequel leur domaine est circonscrit. Les intérêts standard imputables ne sont pas non plus hiérarchisés de manière univoque. L'acteur individuel peut s'identifier à eux de façon sélective et les hiérarchiser à sa manière. Lorsqu'ils ont la forme de préférences stables, ils deviennent des éléments d'une orientation propre à l'acteur et valable dans toutes les situations (figure 2). Par exemple, si un acteur corporatif considère que la croissance est un but plus important que la protection de son domaine, il accordera plus de place à cet intérêt dans ses actions, toutes autres choses égales par ailleurs, qu'un acteur ayant l'attitude inverse. L'intérêt qui gouverne l'action dans chaque cas particulier dépend en fin de compte des particularités de la situation: les intérêts activés lorsque les ressources viennent à manquer ne sont pas les mêmes que lorsque le domaine ou même l'existence de l'acteur sont menacés.

L'influence des attentes normatives sur l'action peut être représentée dans un modèle par niveaux, tout comme la séquence des "impératifs

54. Schimank (U.), "Politische Steuerung in der Organisationsgesellschaft am Beispiel der Forschungspolitik ", in Zapf (W.), ed., Die Modernisierung moderner Gesellschaften. Verhandlungen des 25. Deutschen Soziologentages in Frankfurt am Main 1990. Francfort/Main, Campus, 1991. 
fonctionnels/préférences générales des acteurs/intérêts et gouvernent, en fonction de la situation, les intérêts gouvernant l'action ». Bien que les attentes normatives soient formulées en partie sous la forme de valeurs ou de qualités valables dans l'ensemble du système, elles s'adressent souvent spécifiquement à ceux qui occupent des positions sociales précises et sont comprises dans le contexte institutionnel. Au premier niveau, les attentes normatives restent des consignes extérieures à l'acteur, bien que celui-ci puisse les intérioriser sous forme d'orientations normatives stables ${ }^{55}$. Là encore, c'est la situation concrète de l'action qui active sélectivement ces orientations, aussi bien pour les acteurs corporatifs que pour les acteurs individuels. Les attentes normatives s'adressent aussi aux acteurs corporatifs, puisqu'elles englobent notamment les tâches qu'ils doivent accomplir (objet de l'organisation) et les moyens admis pour leur accomplissement.

Le concept de "comportement approprié » de March et Olsen 56 réunit l'action déterminée par la norme et l'action déterminée par l'identité. Si nous militons pour que l'identité propre soit conservée et confirmée comme référent autonome pour le choix des options de l'action, c'est parce que l'identité de l'acteur ne se résume pas à des normes internalisées. Si l'on entend par identité une image (simplifiée) de soi, contenant des aspects liés à l'être et d'autres liés au comportement ${ }^{57}$, alors certaines particularités (le sexe de l'individu, par exemple) et activités (la peinture chez l'artiste, la production d'appareils électroménagers pour une entreprise) font aussi partie du cour de l'identité propre. La figure 2 illustre notre propos. L'identité (consciente) d'un acteur peut inclure des intérêts (par exemple la revendication d'un domaine précis) et des orientations normatives (par exemple la définition d'un comportement respectueux de l'environnement), qui se situent alors à la croisée des deux colonnes du schéma, ainsi que d'autres caractéristiques essentielles qui ne peuvent être subsumées (par exemple le caractère étatique ou d'intérêt général d'une organisation, le sexe ou l'appartenance ethnique d'un individu). En tant que référent de l'action, l'identité est transversale aux normes et aux intérêts et les transcende. Comme l'illustre le comportement de différents acteurs dans le domaine de

55. Les sociologues, en particulier, insistent sans relâche sur l'opposition entre l'action rationnelle par rapport à un but, gouvernée par l'intérêt, et l'action à détermination normative, et opposent l'Homo sociologicus, déterminé par la société, et l'Homo áconomicus, acteur rationnel autodéterminé. Il serait toutefois erroné de formuler une opposition de principe entre valeurs et intérêts : l'autonomie et l'autodétermination, par exemple, sont les deux à la fois. A l'analyse, la dichotomie conceptuelle entre normes (valeurs) et intérêts est beaucoup plus floue puisqu'elle n'est pas facilement réductible à une opposition entre le devoir (exogène) et le vouloir (endogène).

56. March (J.), Olsen (J.), Rediscovering Institutions. The Organisational Basis of Politics, New York, Free Press, 1989.

57. Cette dimension apparaît déjà chez Parsons (T.), The Social System, Glencoe, Free Press, 1951, p. 63-64. 
la politique de la recherche lors de l'unification allemande ${ }^{58}$, la conservation de l'identité propre d'un acteur peut susciter de sa part des comportements différents de ceux qui serviraient ses propres intérêts (organisationnels) en fonction de la situation ou qui répondraient aux attentes normatives de ses partenaires. Le risque d'apparition de conflits entre différentes orientations montre à quel point ce troisième référent à l'action est autonome. Le cadre institutionnel, par le biais des normes et des missions auxquelles s'identifie l'acteur, imprime sa marque sur l'identité. Mais les identités ont aussi d'autres racines liées, pour un individu, à son histoire et souvent à sa socialisation, ou bien, pour les acteurs corporatifs, à sa culture d'organisation, qui s'est constituée avec le temps, voire à sa "corporate identity ", qui a été modelée activement.

Outre les composantes cognitives et motivationnelles de l'orientation de l'action, il convient de souligner l'importance, pour l'analyse des processus $\mathrm{d}^{\prime}$ interactions stratégiques, d'un aspect relationnel rarement abordé dans les théories classiques de l'action: les orientations issues des interactions. Il s'agit ici d'interprétations (typifiées) de la relation entre plusieurs acteurs. Les principales orientations des interactions définissent la relation comme "hostile" (la perte subie par l'autre apparaît comme un gain pour soi), "compétitive" (différence entre le gain de l'autre et le sien propre), "égoïste-rationnelle" (seul le propre profit compte) ou "coopérative" (recherche d'un profit mutuel ${ }^{59}$ ). Dans certains contextes, un certain type d'orientation des interactions peut correspondre à une attente normative. Chez les individus, une telle orientation peut aussi constituer un trait de personnalité, lié par exemple à la socialisation ou à la culture ${ }^{60}$, et être ainsi intégrée à l'image de soi, c'est-à-dire à l'identité.

Il va de soi que, dans bien des situations, différents points de vue guidant l'action coexistent, chacun pouvant, pris isolément, motiver différemment l'action. Pour comprendre la manière dont les acteurs peuvent régler les conflits d'orientation potentiels, il faut recourir à une théorie de l'action. S'il n'est pas indispensable, pour expliquer les macrophénomènes, d'expliquer comment est apparue une orientation donnée, en revanche, il importe de savoir quelle était l'orientation gouvernant l'action des acteurs centraux. A cet égard, une thèse essentielle de la théorie de l'action postule que (contrairement à ce que prétend la théorie économique) les acteurs n'ont pas

58. Maynt\% (R.), Deutsche Forschung im Einigungsprozess. Die Transformation der Akndemic der Wissenschaften der DDR 1989 bis 1992, Francfort/Main, Campus, 1994.

59. Scharpf (F.W.), "Decision Rules, Decision Styles, and Policy Choices ", Journal of Theoretical

Politics, 1, 1989 ; Scharpf (F.W.), "Positive und negative Koordination in Verhandlungssystemen ", in Heritier (A.), ed., Policy Analyse. Kritik und Neuorientierung, Politische Vierteljahresschrift, Sonderheft 24, Opladen, Westdeutscher Verlag, 1994.

60. Liebrand (W.), van Rung (G.), "The Effects of Social Motives on Behavior in Social Dilemmas in Two Cultures ", Journal of Experimental Social P'sychology, 21, 1985. 
tendance à hiérarchiser durablement leurs différents critères, ni même à s'orienter selon une fonction agrégée de l'utilité réunissant les différents critères et tenant compte de leur pondération. Le facteur essentiel est ici la situation de l'action, à laquelle nous consacrerons le paragraphe suivant.

\section{Situations de l'action}

Les acteurs agissent toujours dans des situations concrètes. Lorsqu'on parle de la situation dans le cadre de la théorie de l'action, on entend habituellement par-là l'environnement d'un acteur individuel, au sens des circonstances sociales et non sociales pertinentes pour l'action. Pour l'analyse des processus dans les systèmes d'interorganisation sectoriels (ou les réseaux politiques), il est pourtant souvent important de prendre en compte les situations qui concernent, du point de vue de l'observateur, une pluralité d'acteurs ou un secteur entier. On parle par exemple de la situation du bâtiment ou de celle de l'agriculture. Reste à savoir dans quelle mesure on peut appliquer telles quelles à des secteurs entiers des catégories définies suivant le modèle de l'acteur individuel et de son environnement, pour caractériser les situations pertinentes pour l'action.

La pertinence des situations pour l'action relève, d'une part, des stimuli qu'elles apportent, et d'autre part des possibilités d'action qu'elles offrent aux acteurs. Une situation peut provoquer l'action parce qu'elle confronte le ou les acteurs à un problème ou parce qu'elle leur offre, au contraire, des opportunités particulières. En la matière, la perspective d'une perte semble motiver plus fortement que des gains potentiels ${ }^{61}$, mais des menaces très lourdes peuvent aussi paralyser la capacité à agir. Dans la perspective du choix rationnel, on distingue, en fonction du degré de risque de perte, des situations à coût élevé et d'autres à faible coût, qui motivent des réactions différentes de la part des acteurs ${ }^{62}$. L'action peut toutefois être aussi provoquée par un motif lié à la régulation institutionnelle : c'est ainsi que sont définies les circonstances dans lesquelles un policier doit intervenir, un parti politique, présenter des candidats ou le parlement, débattre d'un projet de loi.

En provoquant l'action, les situations activent de manière sélective certains aspects de l'orientation de l'action présente à l'état latent. Une situation menaçant l'existence de l'acteur active surtout ses intérêts de survie. On peut supposer que les valeurs et les normes gouvernent l'action davantage dans les situations où l'acteur n'est pas directement menacé. De même, selon la situation, un même acteur se sentira concerné en tant que membre d'une famille, d'une organisation ou dans ses fonctions professionnelles. Les

61. Kahneman (D.), Tversky (A.), “Choices, Values, and Frames ", American Psychologist, $39,1984$. 62. Zintl (R.), " Der Homo Oeconomicus : Ausnahmeerscheinung in jeder Situation oder Jedermann in Ausnahmesituationen? ", Analyse $\mathcal{E}$ Kritik, 11, 1989. 
événements qui affectent non seulement les acteurs individuels, mais aussi de grands systèmes sociaux structurés créent un profil précis de répercussions. Ils affectent de différente manière les intérêts des acteurs en présence et définissent pour certains (mais pas tous) des tâches à exécuter. La réunification allemande est un exemple particulièrement spectaculaire d'un tel événement ayant généré des "profils de répercussions " différents selon les secteurs de politique. Le profil de répercussions permet de tirer les premières conclusions des orientations de l'action probablement activées. Ceci étant dit, les répercussions d'un événement affectant un pan entier de la société n'ont pas nécessairement la même intensité dans toutes les subdivisions du système et, surtout, ne sont pas les mêmes dans chacune de celles-ci. C'est ce qu'illustre par exemple l'évolution des grands instituts de recherche ouest-allemands dans la situation créée par la réunification 63 .

Les situations ne font pas que provoquer l'action: elles offrent aussi des options d'action déterminées en partie par les institutions, mais aussi par de nombreux facteurs non imputables au contexte institutionnel. La situation comprend ainsi les ressources effectivement disponibles, qui ne recoupent pas nécessairement celles affectées par l'institution. Les alternatives offertes par la situation décrivent, dans une certaine mesure, le jeu au sein duquel évolue l'acteur. Le choix d'une stratégie dépend aussi des caractéristiques de la situation, telles qu'elles ont été élaborées spécifiquement par la théorie des organisations, à savoir la "libéralité " de l'environnement organisationnel (sa richesse en ressources disponibles et en alternatives), sa stabilité, sa variabilité (voire sa turbulence) et sa complexité64. Toutes les caractéristiques propres à une situation directement pertinentes pour l'action sont des aspects environnementaux perçus. Les ressources doivent être perçues comme disponibles. Selon ses aptitudes cognitives, l'acteur trouvera une situation donnée plus ou moins complexe. Il existe toutefois, outre la situation que l'acteur perçoit et qui influence ainsi directement son action, une situation "réelle" hypothétique, perçue par les observateurs disposant de plus d'informations. Cette situation "réelle " codétermine le succès ou l'échec de l'action: un cavalier prenant un lac gelé couvert de neige pour une prairie va s'y élancer au trot, et c'est l'épaisseur de la glace qui décidera s'il parviendra sur l'autre rive ou brisera la glace et s'enfoncera dans l'eau.

63. Stucke (A.), " German National Research Centers under Political Pressure : Interference between Different Levels of Actors ", in Schimank (U.), Stucke (A.), eds, Coping with Trouble'. Hoz' Science Reacts to Political Disturbances of Research Conditions, Francfort/Main, Campus, 1994.

64. Child (J.), "Organization Structure, Environment, and Performance. The Role of Strategic Choice ", Sociology, 6, 1972. 


\section{L'action dans les constellations d'acteurs}

La gestion des problèmes qui se posent au niveau sectoriel ou de l'ensemble de la société ne dépend quasiment jamais d'un seul acteur. Elle est habituellement l'objet d'interactions au sein d'une constellation d'acteurs disposant pour agir d'options interdépendantes. Dans ces conditions, si le résultat global ne peut être imputé à un seul acteur, il ne représente pas non plus simplement l'effet agrégé d'une pluralité d'actions individuelles sans lien : il est le résultat d'une interdépendance complexe d'actions liées entre elles. Les possibilités de décrire la structure des sous-systèmes sociaux (ou des secteurs) ne manquent pas. On peut utiliser, par exemple, le modèle prédominant de la division du travail ou de la répartition socio-économique des opportunités (stratification sociale). Dans l'institutionnalisme centré sur les acteurs, au contraire, ce sont les différents modes de coordination sociale de l'action qui se trouvent au premier plan, débattus souvent aujourd'hui sous le nom de gouvernance, emprunté à l'analyse économique du coût des transactions. La typologie courante est partie de l'opposition entre le marché et la hiérarchie pour s'élargir progressivement (en intégrant par exemple la solidarité, les réseaux, les associations) ; elle souffre de la pluridimensionnalité (généralement implicite) de la classification sous-tendant ces différents types. La difficulté vient surtout de la démarcation entre modèles structurels et méthodes de coordination : le "réseau », par exemple, est plutôt une structure et la "solidarité " une forme de coordination. Mais dès lors qu'il est question de structures, il est question de la mise en relation des unités : il n'est donc logiquement pas possible de concevoir les structures et les formes $\mathrm{d}$ 'interaction de manière totalement indépendante. Ainsi, d'un point de vue purement conceptuel, la structure en réseau exclut en elle-même un mode de coordination hiérarchique, tandis que l'adaptation unilatérale est le seul mode possible de coordination de l'action dans une population composée d'éléments non liés et n'interagissant pas. Il est en outre toujours important de ramener les divers concepts au même niveau du système, en l'occurrence le secteur ou le sous-système social.

Si l'on tient à l'approche analytique (et non purement phénoménologique), la complexité excessive des structures réelles oblige à une réduction simplificatrice. Nous avons choisi, comme principales formes abstraites de la coordination sociale de l'action, l'adaptation unilatérale ou mutuelle, la négociation, la concertation et la décision hiérarchique. La dimension analytique de ces formes de gouvernance tient au degré d'autonomie individuelle des acteurs ou de leur capacité à agir collectivement ${ }^{65}$. A une extrémité de l'échelle, la coordination n'est possible que si les acteurs s'adaptent, chacun pour soi (et sans avoir connaissance de l'interdépendance

65. Scharpf (F.W.), Mohr (M.), Efficient Self-Coordination in Policy Networks. A Simulation Study, MPIFG Discussion Paper 94/1, Cologne, MPI fur Gesellschaftsforschung, 1994. 
de leurs actions) aux conditions de leur situation qui ont été influencées par les autres ("coordination écologique" ou "ajustement paramétrique" (parametric adjustment ${ }^{66}$ ). L'étape suivante est l'adaptation mutuelle sans concertation: les acteurs continuent à agir chacun pour soi, mais ils ont désormais connaissance de leur interdépendance et anticipent rationnellement les actions et réactions de leurs partenaires ou adversaires. S'ajoute à cela, en cas de "coordination négative", la prise en compte unilatérale des intérêts (de fait ou de droit) des autres acteurs. L'étape suivante qui correspond à la forme de gouvernance de "marché », est caractérisée par des ententes explicites entre les acteurs, mais limitées dans leur objet et dans le temps et habituellement bilatérales. A l'inverse, les " réseaux" peuvent être caractérisés comme des systèmes de négociation conçus pour durer. Dans la forme subordonnée du "système de négociation forcée ", l'option de retrait est effectivement voire juridiquement interdite aux participants. Ils perdent alors la possibilité d'une action autonome ${ }^{67}$, mais sont encore assurés que la décision ne pourra pas être prise sans leur accord. Mais cette sécurité n'existe plus si - dans une association organisée démocratiquement ou dans des communautés politiques - les décisions engageant la collectivité se prennent par vote à la majorité des membres ou dans des assemblées représentatives. Le rapport entre autonomie individuelle et capacité d'action collective est en outre différent selon que les ressources (financières ou humaines) nécessaires à l'exécution des décisions collectives sont collectivisées ou restent détenues par les membres (ce qui est le cas, par exemple, pour l'application des décisions de grève prises par les syndicats). La capacité d'action collective atteint enfin son maximum si la formation de la volonté et l'accès aux ressources collectivisées sont confiées à une autorité hiérarchique (qui dépend toutefois encore du soutien général).

Comme l'a montré le chapitre précédent, aucun secteur de la société ne peut être entièrement décrit par une seule de ces formes de gouvernance. Ainsi, l'économie des sociétés développées n'est jamais organisée seulement comme un "marché ", ni l'Etat comme une " hiérarchie " cohérente. Dans les secteurs para-étatiques, on retrouve généralement des éléments de tous les types de gouvernance, mélangés en combinaisons complexes, mais souvent aussi des formes de coordination horizontale (réseaux ou systèmes de négociation). La distinction entre les différents types de gouvernance n'est plus alors suffisante, pour rendre compte, par exemple, des différences entre d'une part le mode d'autogestion du secteur de la santé, qui repose en Allemagne sur un système de négociations entre les caisses d'assurance maladie et les fédérations de médecins conventionnés, et d'autre part le système allemand de la recherche, caractérisé, lui aussi, par un niveau

66. Lindblom (C.), The Intelligence of Democracy. Decision-Making through Mutual Adjustment, New York, Free Press, 1965.

67. Kliemt (H.), "The Calculus of Consent after Thirty Years ", Public Choice, 79, 1994. 
important d'autorégulation, ces différences étant pertinentes pour le déroulement des processus de décision réels. L'étendue et la forme de l'autorégulation sectorielle varient au gré des interactions entre les acteurs étatiques et sociaux. De même, les différentes catégories de gouvernance stricto sensu ne conviennent pas pour décrire les structures de décision enchevêtrées, par exemple l'imbrication politique verticale entre le gouvernement fédéral allemand et les Länder, ou entre l'Union européenne et ses Etats membres. Il est pourtant indispensable de caractériser précisément la constellation d'interactions pour expliquer correctement les choix stratégiques des acteurs corporatifs et le résultat de leurs interactions.

Les explications « historiques 68 " ont toujours excellé à retracer les processus d'interaction réels complexes. Dans les sciences sociales théoriques, l'analyse des réseaux (pour la description structurelle de constellations d'acteurs complexes) et la "théorie des jeux" mathématisée (pour l'analyse des interactions et de leurs résultats dans les situations d'interdépendance stratégique) permettent une reconstitution systémique simplifiée et, donc théorisable, des macroprocessus, notamment dans les secteurs hautement organisés et sous forme de réseaux. L'analyse des réseaux permet d'avoir une vision globale des relations durables entre les acteurs, et de les caractériser en fonction de différents éléments analytiques ${ }^{69}$. Tous les acteurs capables d'interaction dans un même champ social ne communiquent, coopèrent ou n'échangent pas nécessairement des ressources avec les autres acteurs, tous les acteurs ne dépendent pas de la même manière des autres et n'influencent pas les autres de la même manière. Pour une bonne part, cette structuration des schémas relationnels est déterminée par les institutions : le règlement intérieur du gouvernement fédéral détermine les relations de cosignature entre les ministères; la succession des réformes des caisses d'assurance maladie renouvelle régulièrement la régulation du tissu des négociations entre les caisses, les médecins, les hôpitaux et les associations professionnelles. Bien des relations importantes (et de nombreux aspects de toutes les relations) restent toutefois informelles; elles sont déterminées par des dépendances concrètes, des convergences d'intérêts et d'objectifs ou encore simplement par l'expérience d'une confiance réciproque. Les méthodes de l'analyse des réseaux (analyse multidimensionnelle, modélisation par blocs et théorie des graphes) permettent de faire ressortir les schémas structurels latents et, pour autant que les données disponibles permettent cette interprétation, les potentiels d'influence. La représentation des schémas de relations durables entre les

68. Lübbe (H.), "Was heiBt "Das kann man nur historisch erklaren" ? Zur Analyse der Struktur historischer Prozesse ", in Lübbe (H.), Fortschritt als Orientierungsproblem. Aufklärung in der Gegenwart, Fribourg, Rombach, 1975.

69. Scott (J.), Social Network Analysis. A Handbook, Londres, Sage 1991 ; Pappi (F.) et al., eds, Methoden der Netzwerkanalyse. Techniken der empirischen Sozialforschung 1, Munich, Oldenbourg, 1987. 
acteurs permet de donner aux études de cas un fondement structurel qui ne pourrait être obtenu autrement. L'utilité de l'analyse des réseaux est toutefois limitée parce qu'elle est difficilement exploitable au niveau théorique et que la fiabilité empirique de ses données est médiocre. Ainsi, l'appartenance aux mêmes groupes de travail attestée par les listes officielles, ou encore l'existence de liens d'information qui peut être révélée des entretiens, peuvent signifier beaucoup ou peu de choses. La fréquence des contacts n'est pas non plus nécessairement un bon indicateur de l'intensité d'une relation, ni de sa solidité dans les situations critiques.

La théorie des jeux fournit quant à elle des catégories utiles pour analyser plus précisément les caractéristiques des réseaux d'acteurs particulièrement pertinentes pour l'étude des processus de régulation (Steuerung) et de prise de décision collective. Si l'analyse des réseaux, qui se concentre sur des structures relativement durables, permet tout au plus de renseigner sur la qualité moyenne des relations et, par conséquent, sur la structure des opportunités pour des interactions particulières, la force de la théorie des jeux réside précisément dans l'analyse individuelle des interactions. Les deux méthodes sont donc complémentaires, bien qu'elles aient été mises en œuvre presque toujours séparément jusqu'à présent ${ }^{70}$. La théorie des jeux permet de typifier la structure de conflits résultant des préférences des acteurs. Cependant, la simple caractérisation théorique de jeux de type deux fois deux produit déjà 78 constellations différentes ${ }^{71}$, et toute classification complète de constellations à trois acteurs ou plus serait inexploitable parce qu'excessivement complexe. Même la tentative de ramener le potentiel de conflit de tous les types de structures de préférences à une seule dimension uniforme ${ }^{72} \mathrm{~s}^{\prime}$ est heurtée à des difficultés théoriques, si bien qu'on ne peut que se rabattre sur des classifications grossières, comme celle proposée par Zürn ${ }^{73}$ pour analyser les constellations en politique internationale ${ }^{74}$. En conséquence, nous distinguons, selon la congruence ou l'incongruence des préférences, des jeux de coordination purs, des jeux de coordination avec

70. Scharpf (F.W.), ed., Games in Hierarchies and Networks. Analytical and Empirical Approaches to the Study of Governance Institutions, Francfort/Main, Campus, 1993.

71. Rapoport (A.), Guyer (M.), Gordon (D.), The $2 \times 2$ Game, Ann Arbor, University of Michigan Press, 1976.

72. Axelrod (R.), ed., Conflict of Interest. A Theory of Divergent Goals with Applications to Politics, Chicago, Markham, 1970.

73. Zürn (M.), Interessen und Institutionen in der internationalen Politik. Grundlegung und Anwendungen des situationsstrukturellen Ansatzes, Opladen, Leske Budrich, 1992.

74. L. Greenhalgh et R. Kramer ont mis au point une classification à huit niveaux, allant de la symbiosis à l'enmity, en passant par les niveaux de alliance, conditional cooperation, instrumental interdependence, peaceful coexistence, benign competition et rivalty, sans toutefois donner une définition explicite de ces catégories (intuitivement plausibles) selon la théorie des jeux. Greenhalgh (L.), Kramer (R.), "Strategic Choice in Conflicts. The Importance of Relationships ", in Kahn (R.), Zaid (M.), eds, Organizations and Nation States. New Perspectives on Conflict and Cooperation, San Francisco, Jossey-Bass, p. 184. 
conflits de répartition, des jeux de dilemmes et des jeux de conflits purs. Dans le premier cas, il existe une solution coopérative que tous les participants préfèrent au statu quo. Dans le deuxième cas, il y a deux ou plusieurs solutions coopératives, chacune privilégiant certains participants et en désavantageant d'autres; la stabilité de la solution coopérative finalement choisie est cependant ici assurée. Il en va autrement dans les situations de dilemmes, où l'intérêt commun pour une solution coopérative est menacé par la tentation d'exploiter les partenaires disposés à coopérer. Dans les situations purement conflictuelles, enfin, le résultat escompté d'une confrontation est plus avantageux, pour l'une au moins des parties, que la meilleure solution que les participants pourraient trouver en commun.

Les tentatives d'explication recourant aux catégories de la théorie des jeux sont souvent compliquées par l'existence d'interactions à plusieurs niveaux ${ }^{75}$ ou de jeux couplés (two-level games ou nested games, $)^{76}$. La constellation prototypique dans ce cas sera les négociations intergouvernementales dont le résultat doit être ratifié par chaque parlement national, mais on rencontre des conditions structurellement analogues dans tous les systèmes d'interaction imbriqués. C'est le couplage entre les interactions externes et internes qui est problématique, puisqu'il peut créer une divergence dans les orientations de l'action et, de ce fait, risque de diminuer soit l'aptitude à résoudre les problèmes par la négociation externe, soit l'intégrité de la formation de la volonté interne ${ }^{77}$.

Les constellations d'interactions (jeux) entre les acteurs corporatifs sont en grande partie influencées par les institutions dans leurs aspects importants, mais pas au point de pouvoir être définies uniquement en fonction de critères institutionnels. Ainsi, bien que les «joueurs" soient constitués institutionnellement comme des acteurs corporatifs, leur participation à un processus décisionnel donné, leurs perceptions, leurs objectifs d'action et leurs relations aux autres acteurs sont aussi influencés par des facteurs non institutionnels. Les critères institutionnels ne sont pas seuls à déterminer si les acteurs se situent dans un jeu de coordination, de dilemme, voire de conflit: les interprétations subjectives de la situation réelle ont aussi leur importance, de même que les orientations gouvernant effectivement

75. Benz (A.), "Mehrebenen-Verflechtung: Verhandlungsprozesse in verbundenen Entscheidung sarenen ", in Benz (A.), Scharpf (F.W.), Zintl (R.), Horizontale Politikverflechtung. Zur Theoric von Verhandlungssystemen, Francfort/Main, Campus, 1992.

76. Putnam (R.), "Diplomacy and Domestic Politics. The Logic of Two-level Games", International Organization 42, 1988 ; Tsebelis (G.), Nested Games. Rational Choice in Comparative Politic, Berkeley, University of California Press, 1990.

77. Scharpf (F.W.), "Versuch uber Demokratie im verhandelnden Staat ", in Czada (R.), Schmidt (M.), eds, Verhandlungsdemokratie, Interessenvermittlung, Regierbarkeit. Festschrift fïr Gerhard Lehmbruch, Opladen, Westdeutscher Verlag, 1993. 
l'action $^{78}$. Comme l'analyse des réseaux, la modélisation selon la théorie des jeux a elle aussi ses limites. Cette théorie mathématique formule des hypothèses irréalistes quant au niveau d'information et à la capacité de traitement de l'information des joueurs modélisés, ce qui conduit les théoriciens des jeux eux-mêmes à se montrer sceptique quant à l'adaptation de leurs modèles à l'explication d'interactions empiriquement observées entre des acteurs réels ${ }^{79}$. Ceci étant, il est possible de construire des modèles selon la théorie des jeux (ou selon la théorie des contrats, sur la base de la théorie des jeux), qui sont applicables à notre type de problématique ${ }^{80}$.

\section{L'institutionnalisme centré sur les acteurs, entre " parcimonie " (parsimony) et « diversité nécessaire » (requisite variety)}

Comme nous l'avons exposé longuement plus haut, l'institutionnalisme centré sur les acteurs risque de devenir excessivement complexe en raison de l'intégration des perspectives institutionnalistes et des théories de l'action, et d'imposer en pratique une sorte de reconstruction historique. Ce problème est fondamentalement insoluble, mais il peut être simplifié en respectant quelques règles pragmatiques de recherche. Le problème central, à savoir la possibilité d'imputer ou non une action observable au contexte institutionnel ou à l'un des nombreux facteurs non institutionnels, peut être simplifié par une hiérarchisation analytique. Même lorsqu'ils reconstruisent des évolutions historiques uniques, les sociologues ne recherchent pas une explication complète, une reproduction à l'identique de la réalité, mais une simplification exacte. En d'autres termes, ils tentent, en introduisant le moins d'informations empiriques ad hoc possible, de résoudre le plus

78. Ces orientations peuvent parfois changer rapidement. Ainsi, dans une étude de cas, la réussite d'un projet de recherche industrielle mixte, soutenu par le gouvernement, est imputée au passage d'une orientation compétitive à une orientation coopérative dans les interactions, ce changement étant motivé par une nouvelle interprétation d'une situation objectivement inchangée. Lütz (S.), Die Steuerung industrieller Forschungskooperation. Funktionsweise und Erfolgsbedingungen des staatlichen Forderinstrumentes Verbundforschung, Francfort/Main, Campus, 1993.

79. Binmore (K.), "Why Game Theory 'Doesn't Work' ", in Bennett (P. O.), ed., Analysing Conflict and its Resolution, Oxford, Clarendon Press, 1987.

80. Ceci se vérifie notamment lorsque l'on tient compte non seulement de la théorie des jeux simultanés dans des conditions de " connaissances partagées, mais aussi des modèles de la théorie évolutionniste des jeux et des jeux à informations incomplètes, dans lesquels les exigences d'information et de capacité de traitement de l'information des acteurs sont beaucoup moins strictes ". Cf. Benz (A.), Scharpf (F.W.), Zintl (R.), Horizontale Politikverflechtung. Zur Theorie a'on Verhandlungssystemen, Francfort/Main, Campus, 1992 ; Ryll (A.), Die Spieltheorie als Instrument der Gesellschaftsforschung, MP1FG Discussion Paper 89/10, Cologne, MPI für Gesellschaftsforschung, 1989 ; Scharpf (F.W.), Mohr (M.), Efficient Self-Coordination..., op. cit. ; Werle (R.), "Rational Choice und rationale Technikentwicklung. Einige Dilemmata der Technikkoordination Theory ", in Halfmann (J.), et al., eds, Technik und Gesellschaft, Jahrbuch 8 : Theoriebausteine der Techniksoziologie, Francfort/Main, Campus, 1995. 
"d'énigmes " possible présentes dans chaque problématique. Ils peuvent pour cela s'aider d'une variante institutionnaliste de la règle " $d$ 'abstraction décroissante ${ }^{81}$ ", qui est la suivante: on ne doit pas expliquer par rapport aux acteurs ce qui peut être expliqué par les institutions, et les explications se rapportant aux acteurs doivent partir d'hypothèses simplificatrices, qui ne seront vérifiées empiriquement que s'il n'est pas possible d'expliquer les actions observables autrement. L'approche institutionnelle réduit ainsi considérablement le volume d'informations nécessaire à une explication satisfaisante. En effet, puisque le contexte institutionnel codétermine non seulement les structures relationnelles et les motifs d'interaction, mais aussi les orientations de l'action, on en sait déjà long sur les acteurs lorsque l'on connaît leur contexte: non seulement leurs obligations d'action ou d'omission, les options d'action autorisées et leurs ressources légitimes, mais aussi leurs intérêts organisationnels propres, souvent leur orientation caractéristique dans les interactions (comme dans le cas de la relation "compétitive » entre le gouvernement et l'opposition dans les régimes parlementaires) et leurs tendances perceptives (la Bundesbank est plus sensible aux risques d'inflation que le ministère du Travail). Il faut une situation de grand bouleversement, comme ce fut le cas en Allemagne de l'Est pendant la réunification, pour que les structures institutionnelles perdent leur pouvoir d'orientation. Dans ce cas, un rôle plus important est dévolu, par la force des choses, aux facteurs de situation et même aux spécificités individuelles.

Dans les processus sectoriels de régulation et d'autorégulation, la détermination empirique des orientations spécifiques aux acteurs n'est pas nécessaire si les tâches affectées par l'institution et les intérêts organisationnels propres supposés suffisent à expliquer l'action observable. Une identification empirique des motivations effectives ne devient nécessaire que si ces éléments ne concordent manifestement pas avec les intérêts supposés dans une situation donnée. De même, les tentatives d'explication s'appuieront, autant que possible, sur la supposition de cognitions "réalistes " et conventionnelles, et le recours aux méthodes de recherche poussées sera réservé aux cas où l'action observable semble indiquer l'existence de distorsions dans la perception. Cette approche correspond à l'utilisation weberienne des modèles d'action rationnelle comme type d'action conforme, qui sert d'hypothèse dans les analyses empiriques et oriente éventuellement vers les autres explications possibles.

Il est quasiment impossible d'appliquer à une étude empirique une méthode de recherche intégrant systématiquement un contexte institutionnel à

81. Lindenberg (S.), «Die Methode der abnehmenden Abstraktion. Theoriegesteuerte Analyse und empirischer Gehalt ", in Esser (H.), Troitzsch (K.), eds, Modellierung sozialer Prozesse, Bonn, Informationszentrum Sozialwissenschaften, 1991. 
plusieurs niveaux, des acteurs individuels et corporatifs, les orientations de ceux-ci, leurs perceptions, relations et interactions. Les études de l'Institut Max-Planck für Gesellschaftsforschung n'utilisent donc généralement que des segments précis de cette grille analytique complexe : ce sont parfois les orientations de l'action et leurs déterminations empiriques qui ont été mises en avant 82 ; ailleurs, on a employé pour les orientations de l'action des postulats dérivés des intérêts organisationnels propres supposés ${ }^{83}$. Dans certaines études, les acteurs ont été explicitement intégrés à différents niveaux ${ }^{84}$. Dans $d^{\prime}$ 'autres, les organisations ont été traitées comme des acteurs unitaires. Ces raccourcis se retrouvent dans les chapitres qui suivent. Ils ont en commun non seulement de se rapporter à des aspects d'une thématique globale (régulation et autoorganisation dans les secteurs paraétatiques), mais aussi de se concentrer sur les interactions entre les acteurs (corporatifs et autres) dans des constellations interdépendantes. C'est cette détermination de l'objet de l'étude qui fait le lien entre les différentes contributions, bien plus que le traitement plus ou moins complet des catégories analytiques élaborées à cette fin. Quoi qu'il en soit, les études empiriques bénéficient de la connaissance de toute la complexité d'une approche de recherche à la fois institutionnaliste et centrée sur les acteurs.

82. Mayntz (R.), Neidhardt (F.), "Parlmentskultur. Eine empirisch-explorative Studie ", Zeitschirift für Parlamentsfragen, 20, 1989 ; Derlien (H.-U.), Mayntz (R.), " Party Patronage and Politicization of the West German Administrative Elite 1970-1987. Toward Hybridization ? ", Gozernance, 2, 1989.

83. Par exemple Hohn (H.-W.), Schimank (U.), Konflikte und Gleichgewichte im Forschungssystem. Akteurkonstellationen und Entwicklungpfade in der außeruniversitären Forschung, Francfort/Main, Campus, 1990.

84. Wasem (J.), « Niederlassung oder Poliklini ", art. cité ; Schimank (U.), Hochschulforschung..., op. cit. ; Mayntz (R.), Deutsche Forschung..., op. cit. 